\title{
THE BEST POSSIBLE UNIFICATION FOR ANY COLLECTION OF PHYSICAL THEORIES
}

\author{
ROBERT A. HERRMANN
}

Received 1 July 2003

\begin{abstract}
We show that the set of all finitary consequence operators defined on any nonempty language is a join-complete lattice. This result is applied to various collections of physical theories to obtain an unrestricted standard supremum unification. An unrestricted hyperfinite ultralogic unification for sets of physical theories is also obtained.
\end{abstract}

2000 Mathematics Subject Classification: 03G10, 03H10, 03B80.

1. Introduction. A restricted hyperfinite ultralogic unification is constructed in [5]. The restrictions placed upon this construction were necessary in order to relate the constructed ultralogic directly to the types of ultralogics used to model probability models [6]. In particular, the standard collections of consequence operators are restricted to a very special set of operators $H_{X}$, where $X$ is itself restricted to the set of all significant members of a language $\Lambda$. In this paper, all such restrictions are removed. For readers convincement, some of the introductory remarks that appear in [5] are repeated. Over seventy years ago, Tarski [8, pages 60-109] introduced consequence operators as models for various aspects of human thought. There are two such mathematical theories investigated, the general and the finitary consequence operators [2]. Let $L$ be a nonempty language, $\mathscr{P}$ the power set operator, and $\mathscr{F}$ the finite power set operator. There are three cardinality-independent axioms.

DEFINITION 1.1. A mapping $C: \mathscr{P}(L) \rightarrow \mathscr{P}(L)$ is a general consequence operator (or closure operator) if for each $X, Y \in \mathscr{P}(L)$,

(1) $X \subset C(X)=C(C(X)) \subset L$;

(2) if $X \subset Y$, then $C(X) \subset C(Y)$.

A consequence operator $C$ defined on $L$ is said to be finitary (finite) if it satisfies

(3) $C(X)=\bigcup\{C(A) \mid A \in \mathscr{F}(X)\}$.

REMARK 1.2. The above axioms (1), (2), and (3) are not independent. Indeed, (1) and (3) imply (2). Clearly, the set of all finitary consequence operators defined on a specific language is a subset of the set of all general operators. The phrase "defined on $L$ " means formally defined on $\mathscr{P}(L)$.

All known scientific logic systems use finitely many rules of inference and finitely many steps in the construction of a deduction from these rules. Hence, as shown in [5], the consequence operator that models such theory-generating thought processes is a finitary consequence operator. Although many of the results in this paper hold for 
the general consequence operator, we are only interested in collections of finitary consequence operators. Dziobiak [1, page 180] states Theorem 2.10 below. However, the statement is made without a formal proof and is relative to a special propositional language. Theorem 2.10 is obtained by using only basic set-theoretic notions and Tarski's basic results for any language. Further, the proof reveals some interesting facts not previously known. Unless noted, all utilized Tarski results [8, pages 60-91] are cardinality independent.

\section{The lattice of finitary operators}

DEFINITION 2.1. In all that follows, any set of consequence operators will be nonempty and each is defined on a nonempty language. Define the relation $\leq$ on the set $\mathscr{b}$ of all general consequence operators defined on $L$ by stipulating that for any $C_{1}, C_{2} \in \mathscr{C}$, $C_{1} \leq C_{2}$ if for every $X \in \mathscr{P}(L), C_{1}(X) \subset C_{2}(X)$.

Obviously, the relation $\leq$ is a partial order contained in $\mathscr{b} \times \mathscr{b}$. Our standard result will show that for the entire set of finitary consequence operators $\mathscr{C}_{f} \subset \mathscr{C}$ defined on $L$, the structure $\left\langle\mathscr{C}_{f}, \leq\right\rangle$ is a lattice.

DEFINITION 2.2. Define $I: \mathscr{P}(L) \rightarrow \mathscr{P}(L)$ and $U: \mathscr{P}(L) \rightarrow \mathscr{P}(L)$ as follows: for each $X \subset L$, let $I(X)=X$ and let $U(X)=L$.

Notice that $I$ is the lower unit (the least element) and $U$ the upper unit (the greatest element) for $\left\langle\mathscr{C}_{f}, \leq\right\rangle$ and $\left\langle\mathscr{C}_{,} \leq\right\rangle$.

Definition 2.3. Let $C \in \mathscr{C}$. A set $X \subset L$ is a $C$-system or simply a system if $C(X) \subset X$ and, hence, if $C(X)=X$. For each $C \in \mathscr{C}$, let $\mathscr{Y}(C)=\{X \mid(X \subset L) \wedge(C(X)=X)\}$.

Since $C(L)=L$ for each $C \in \mathscr{C}$, then each $\mathscr{S}(C) \neq \varnothing$.

LEMMA 2.4. For each $C_{1}, C_{2} \in \mathscr{b}, C_{1} \leq C_{2}$ if and only if $\mathscr{S}\left(C_{2}\right) \subset \mathscr{S}\left(C_{1}\right)$.

Proof. Let any $C_{1}, C_{2} \in \mathscr{C}$ and $C_{1} \leq C_{2}$. Consider any $Y \in \mathscr{S}\left(C_{2}\right)$. Then $C_{1}(Y) \subset$ $C_{2}(Y)=Y$. Thus, $C_{1} \in \mathscr{S}\left(C_{1}\right)$ implies that $\mathscr{S}\left(C_{2}\right) \subset \mathscr{S}\left(C_{1}\right)$.

Conversely, suppose that $\mathscr{S}\left(C_{2}\right) \subset \mathscr{Y}\left(C_{1}\right)$. Let $X \subset L$. Then, since, by axiom (1), $C_{2}(X) \in$ $\mathscr{S}\left(C_{2}\right)$, it follows, from the requirement that $C_{2}(X) \in \mathscr{S}\left(C_{1}\right)$, that $C_{1}\left(C_{2}(X)\right)=C_{2}(X)$. But $X \subset C_{2}(X)$ implies that $C_{1}(X) \subset C_{1}\left(C_{2}(X)\right)=C_{2}(X)$, from axiom (2). Hence, $C_{1} \leq C_{2}$ and the proof is complete.

DEFINITION 2.5. For each $C_{1}, C_{2} \in \mathscr{C}$, define the following binary relations in $\mathscr{P}(L) \times$ $\mathscr{P}(L)$. For each $X \subset L$, let $\left(C_{1} \wedge C_{2}\right)(X)=C_{1}(X) \cap C_{2}(X)$ and $\left(C_{1} \vee{ }_{w} C_{2}\right)=\bigcap\{Y \subset L \mid$ $\left.\left(X \subset Y=C_{1}(Y)=C_{2}(Y)\right)\right\}$. For finitely many members of $\mathscr{C}$, the operators $\wedge, \vee_{w}$ are obviously commutative and associative. These two relations are extended to arbitrary $\mathscr{A} \subset \mathscr{b}$ by defining $(\bigwedge \mathscr{A})(X)=\bigwedge \mathscr{A}(X)=\bigcap\{C(X) \mid C \in \mathscr{A}\}$ and $\left(\bigvee_{w} \mathscr{A}\right)(X)=\bigvee_{w} \mathscr{A}(X)=$ $\bigcap\{Y \subset L \mid X \subset Y=C(Y)$ for all $C \in \mathscr{A}\}$ [1, page 178]. Notice that $\bigvee_{w} \mathscr{A}(X)=\bigcap\{Y \subset L \mid$ $(X \subset Y) \wedge(Y \in \bigcap\{\mathscr{S}(C) \mid C \in \mathscr{A}\})\}$.

LEMMA 2.6. Let $\mathscr{A} \subset \mathscr{C}$ (resp., $\left.\mathscr{C}_{f}\right)$ and $\mathscr{S}^{\prime}=\left\{X \mid(X \subset L) \wedge\left(X=\bigvee_{w} \mathscr{A}(X)\right)\right\}$. Then $\mathscr{S}^{\prime}=\bigcap\{\mathscr{Y}(C) \mid C \in \mathscr{A}\}$. 
Proof. By Tarski's [8, Theorem 11(b), page 71], which holds for finitary and general consequence operators, for each $X \subset L$ and $C \in \mathscr{A}, X \subset \bigvee_{w} \mathscr{A}(X)=Y^{\prime} \in \mathscr{S}(C)$. Hence, if $Y^{\prime} \in \mathscr{Y}^{\prime}$, then $\bigvee_{w} \mathscr{A}\left(Y^{\prime}\right)=Y^{\prime} \in \mathscr{S}(C)$ for each $C \in \mathscr{A}$. Thus $\mathscr{S}^{\prime} \subset \bigcap\{\mathscr{Y}(C) \mid C \in \mathscr{A}\}$. Conversely, let $Y \in \bigcap\{\mathscr{S}(C) \mid(C \in \mathscr{A})\}$. From the definition of $\bigvee_{w}, \bigvee_{w} \mathscr{A}(Y)=Y$ and, hence, $Y \in \mathscr{S}^{\prime}$ and this completes the proof.

LEMMA 2.7. Let the nonempty $\mathscr{B} \subset L$ have the property that for each $X \subset L$, there exists some $Y \in \mathscr{B}$ such that $X \subset Y$. Then the operator $C_{\mathscr{B}}$ defined for each $X \subset L$ by $C_{\mathscr{B}}(X)=\bigcap\{Y \mid X \subset Y \in \mathscr{B}\}$ is a general consequence operator defined on $L$.

Proof. Assuming the hypothesis of the Lemma, it is obvious that $C_{\mathscr{B}}: \mathscr{P}(L) \rightarrow \mathscr{P}(L)$ and $X \subset C_{\mathscr{B}}(X)$. Clearly, if $Z \subset X \subset L$, then $C_{\mathscr{B}}(Z) \subset C_{\mathscr{B}}(X)$; and, for each $Y \in \mathscr{B}, X \subset Y$ if and only if $C_{\mathscr{B}}(X) \subset Y$. Hence, $C_{\mathscr{B}}\left(C_{\mathscr{B}}(X)\right)=\bigcap\left\{Y \mid C_{\mathscr{B}}(X) \subset Y \in \mathscr{B}\right\}=C_{\mathscr{B}}(X)$. This completes the proof.

REMARK 2.8. The hypothesis of Lemma 2.7 can be weakened. However, our application does not require such a weakening.

THEOREM 2.9. With respect to the partial order relation $\leq$ defined on $L$, the structure $\left\langle\mathscr{G}, \vee_{w}, \wedge, I, U\right\rangle$ is a complete lattice with upper and lower units.

Proof. Let $\mathscr{A} \subset \mathscr{C}$ and $\mathscr{B}=\bigcap\{\mathscr{S}(C) \mid C \in \mathscr{A}\}$. Since $L \in \mathscr{B}$, then by Lemma 2.7, $\bigvee_{w} \mathscr{A}=C_{\mathscr{B}} \in \mathscr{C}$. Moreover, by Lemmas 2.4 and 2.6, $C_{\mathscr{B}}$ is the least upper bound for $\mathscr{A}$ with respect to $\leq$.

Next, let $\mathscr{B}=\bigcup\{\mathscr{S}(C) \mid C \in \mathscr{A}\}$. For $X \subset L, X \subset C(X)$ for each $C \in \mathscr{A}$. For each $C \in \mathscr{A}$, there does not exist a $Y_{C}$ such that $Y_{C} \in \mathscr{Y}(C), X \neq Y_{C}, Y_{C} \neq C(X)$, and $X \subset Y_{C} \subset C(X)$. Hence, $C_{\mathscr{B}}(X)=\bigcap\{Y \mid X \subset Y \in \mathscr{B}\}=\bigcap\{C(X) \mid C \in \mathscr{A}\}=\bigwedge \mathscr{A}(X)$. Hence, $\bigwedge \mathscr{A} \in \mathscr{C}$ and it is obvious that $\wedge \mathscr{A}$ is the greatest lower bound for $\mathscr{A}$ with respect to $\leq$. This completes the proof.

Although the proof appears in error, Wójcicki [9] stated Theorem 2.9 for a propositional language. In what follows, we only investigate the basic lattice structure for $\left\langle\mathscr{C}_{f}, \leq\right\rangle$.

THEOREM 2.10. With respect to the partial order relation $\leq$ defined on $\mathfrak{b}_{f}$, the structure $\left\langle\mathscr{C}_{f}, \vee_{w}, \wedge, I, U\right\rangle$ is a lattice with upper and lower units.

Proof. It is only necessary to consider two distinct $C_{1}, C_{2} \in \mathscr{C}_{f}$. As mentioned, the commutative and associative laws hold for $\wedge$ and $\vee_{w}$, and by definition, each maps $\mathscr{P}(L)$ into $\mathscr{P}(L)$. In $\langle\mathscr{C}, \leq\rangle$, using Theorem 2.9, axioms (1) and (2) hold for the greatest lower bound $C_{1} \wedge C_{2}$ and for the least upper bound $C_{1} \vee{ }_{w} C_{2}$. Next, we have

$$
\begin{aligned}
\left(C_{1} \wedge C_{2}\right)(X) & =\left(\bigcup\left\{C_{1}(Y) \mid Y \in \mathscr{F}(X)\right\}\right) \cap\left(\bigcup\left\{C_{2}(Y) \mid Y \in \mathscr{F}(X)\right\}\right) \\
& =\bigcup\left\{C_{1}(Y) \cap C_{2}(Y) \mid Y \in \mathscr{F}(X)\right\} \\
& =\bigcup\left\{\left(C_{1} \wedge C_{2}\right)(Y) \mid Y \in \mathscr{F}(X)\right\}
\end{aligned}
$$

and axiom (3) holds and, hence, $C_{1} \wedge C_{2} \in \mathscr{C}_{f}$. Therefore, $\left\langle\mathscr{C}_{f}, \wedge, I, U\right\rangle$ is, at least, a meet semilattice. 
Next, we show by direct means that for each $C_{1}, C_{2} \in \mathscr{C}_{f}, C_{1} \vee w C_{2} \in \mathscr{C}_{f}$. Let (the cardinality of $L)|L|=\Delta$. For each $X_{i} \subset L, i \in \Delta$, let $\mathscr{A}^{\prime}\left(X_{i}\right)=\left\{Y \mid\left(X_{i} \subset Y \in \mathscr{Y}\left(C_{1}\right) \cap\right.\right.$ $\left.\left.\mathscr{S}\left(C_{2}\right)\right) \wedge(Y \subset L)\right\}$. Let $\bigcap\left\{Y \mid Y \in \mathscr{A}^{\prime}\left(X_{i}\right)\right\}=Y_{i}$. By Tarski's [8, Theorem 11, page 71], $X_{i} \subset$ $Y_{i} \in \mathscr{Y}\left(C_{1}\right) \cap \mathscr{Y}\left(C_{2}\right)$, and by definition, $Y_{i}=\left(C_{1} \vee{ }_{w} C_{2}\right)\left(X_{i}\right)$. Hence, $Y_{i} \in \mathscr{A}^{\prime}\left(X_{i}\right)$ and is the least ( $\subset$ ) element. For $X_{i} \subset L$, let $\mathscr{A}^{\prime \prime}\left(X_{i}\right)=\left\{Y \mid\left(C_{1}\left(X_{i}\right) \subset Y \in \mathscr{S}\left(C_{1}\right) \cap \mathscr{S}\left(C_{2}\right)\right) \wedge(Y \subset L)\right\}$. Since $X_{i} \subset C_{k}\left(X_{i}\right), k=1,2$, then $\mathscr{A}^{\prime \prime} \subset \mathscr{A}^{\prime}$. Since $L \in \mathscr{A}^{\prime}\left(X_{i}\right), \mathscr{A}^{\prime}\left(X_{i}\right) \neq \varnothing$. Indeed, let $Y \in \mathscr{A}^{\prime}\left(X_{i}\right)$. Then $X_{i} \subset C_{k}(Y)=Y, k=1$,2. Additionally, $X_{i} \subset C_{1}(Y)=Y$ implies that $X_{i} \subset C_{1}\left(X_{i}\right)=C_{1}\left(C_{1}\left(X_{i}\right)\right) \subset C_{1}\left(C_{1}(Y)\right)=C_{1}(Y)=Y$. Hence, it follows that for any $X_{i} \subset L, \mathscr{A}^{\prime \prime}\left(X_{i}\right)=\mathscr{A}^{\prime}\left(X_{i}\right)$. For fixed $X_{i} \subset L$, let $X_{j} \in \mathscr{F}\left(X_{i}\right)$. Let $Y_{j}$ be defined as above and, hence, $Y_{j}$ is the least element in $\mathscr{A}^{\prime}\left(X_{j}\right)=\mathscr{A}^{\prime \prime}\left(X_{j}\right)$. Consider $\mathscr{D}=\left\{Y_{j} \mid X_{j} \in \mathscr{F}\left(X_{i}\right)\right\}$, and, for $j=1, \ldots, n$, consider $Y_{j} \in \mathscr{D}$ and the corresponding $X_{j} \subset L$. Let $X_{k}=\bigcup\left\{X_{j} \mid j=\right.$ $1, \ldots, n\} \in \mathscr{F}\left(X_{i}\right)$. Then $Y_{k}=\bigcap\left\{Y \mid Y \in \mathscr{A}^{\prime}\left(X_{k}\right)\right\} \in \mathscr{D}$. If $Y \in \mathscr{A}^{\prime}\left(X_{k}\right)$, then $Y \in \mathscr{A}^{\prime}\left(X_{j}\right), j=$ $1, \ldots, n$. Hence, $Y_{j} \subset Y_{k}, j=1, \ldots, n$, implies that $Y_{1} \cup \cdots \cup Y_{n} \subset Y_{k}$. Tarski's theorem [8, Theorem 12, page 71] implies that $Y^{*}=\bigcup\left\{Y_{j} \mid X_{j} \in \mathscr{F}\left(X_{i}\right)\right\} \in \mathscr{Y}\left(C_{1}\right) \cap \mathscr{Y}\left(C_{2}\right)$. Also, by definition, for all $X_{j} \subset L, Y_{j} \in \mathscr{A}^{\prime \prime}\left(X_{j}\right)$ implies that $C_{1}\left(X_{j}\right) \subset Y_{j}$. The fact that $C_{1}$ is finitary yields $C_{1}\left(X_{i}\right) \subset Y^{*}$. Hence, $Y^{*} \in \mathscr{A}^{\prime \prime}\left(X_{i}\right)$. Since $C_{1}\left(X_{j}\right) \subset C_{1}\left(X_{i}\right), X_{j} \in \mathscr{F}\left(X_{i}\right)$, then $\mathscr{A}^{\prime \prime}\left(X_{i}\right) \subset \mathscr{A}^{\prime \prime}\left(X_{j}\right)$. Thus $Y_{j} \subset Y_{i}, X_{j} \in \mathscr{F}\left(X_{i}\right)$. Therefore, $Y^{*} \subset Y_{i}$. But, $Y^{*} \in \mathscr{A}^{\prime \prime}\left(X_{i}\right)$ implies that $Y^{*}=Y_{i}$. Restating this last result, $\bigcup\left\{\left(C_{1} \vee_{w} C_{2}\right)\left(X_{j}\right) \mid X_{j} \in \mathscr{F}\left(X_{i}\right)\right\}=\left(C_{1} \vee_{w}\right.$ $\left.C_{2}\right)\left(X_{i}\right)$ and, therefore, axiom (3) holds for the binary relation $\vee_{w}$, and $\left\langle\mathscr{C}_{f}, \vee_{w}, \wedge, I, U\right\rangle$ is a lattice. This completes the proof.

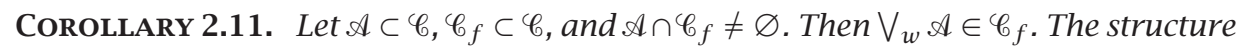
$\left\langle\mathscr{C}_{f}, \vee_{w}, \wedge, I, U\right\rangle$ is a join-complete lattice.

Proof. Simply modify the second part of the proof of Theorem 2.10 by substituting $\cap\{\mathscr{S}(C) \mid C \in \mathscr{A}\}$ for $\mathscr{S}\left(C_{1}\right) \cap \mathscr{S}\left(C_{2}\right)$ and letting $C_{1} \in \mathscr{A} \cap \mathscr{C}_{f}$. This completes the proof.

REMARK 2.12. It is known, since $I$ is a lower bound for any $\mathscr{A} \subset \mathscr{C}_{f}$, that $\left\langle\mathscr{C}_{f}, \vee_{w}, I, U\right\rangle$ is actually a complete lattice with a meet operator generated by the $\vee_{w}$ operator. It appears that the meet operator $\wedge$ for infinite $\mathscr{A}$ need not correspond, in general, to the $\vee_{w}$-defined meet operator. Wójcicki [10] has constructed, for a set of consequence operators $\mathfrak{C}^{\prime}$, an infinite $\mathscr{A} \subset \mathscr{C}^{\prime}$ of finitary consequence operators with some very special properties. However, the general consequence operator defined for each $X \subset L$ by $\bigcap\{C(X) \mid C \in \mathscr{A}\}$ is not a finitary operator. Thus, in general, $\left\langle\mathscr{C}_{f}, \vee_{w}, \wedge, I, U\right\rangle$ need not be a meet complete lattice. This behavior is not unusual. For example, let infinite $X$ have an infinite topology $\mathscr{T}$. Then $\langle\mathscr{T}, \cup, \cap, \varnothing, X\rangle$ is a join-complete sublattice of the lattice $\langle\mathscr{P}(X), \cup, \cap, \varnothing, X\rangle$. The structure $\langle\mathscr{T}, \cup, \varnothing, X\rangle$ is actually complete, but it is not a meet-complete sublattice of complete $\langle\mathscr{P}(X), \cup, \cap, \varnothing, X\rangle$.

3. System-consistent logic systems. Let $\Sigma$ be a nonempty set of science-community logic systems and let $|\cdot|$ denote cardinality. In practice, $|\Sigma| \leq \kappa_{0}$. Each logic system $S_{i} \in \Sigma, i \in|\Sigma|$, is defined on a countable language $L_{i}$ and each $S_{i}$ determines a specific finitary consequence operator $C_{i}$ defined on a language $L_{i}$. At least, by application of the insertion of hypotheses rule [5] for nonempty cardinal $\Delta \leq|\Sigma|$, each member of $\left\{C_{i} \mid i \in \Delta\right\}$ is defined on the language $\bigcup\left\{L_{i} \mid i \in \Delta\right\}$. 
In all that follows, a specific set of logic-system-generated consequence operators $\left\{C_{i} \mid\right.$ $i \in \Delta\}$ defined on a specific set of languages $\left\{L_{i} \mid i \in \Delta\right\}$ will always be considered as trivially extended and, hence, defined by the insertion of hypotheses rule on the set $\bigcup\left\{L_{i} \mid i \in \Delta\right\}$. In general, such a specific set of consequence operators is contained in the lattice of all finitary operators defined on $\bigcup\left\{L_{i} \mid i \in \Delta\right\}$.

A logic system $S^{\prime}$ and its corresponding consequence operator is a trivial extension of a logic system $S$ defined on $L$ where, for a language $L^{\prime} \supset L, S^{\prime}$ is the same as $S$ except that the insertion rule now applies to $L^{\prime}$. The system $S^{\prime}$ and its corresponding consequence operator $C^{\prime}$ is a nontrivial extension if it is extended to $L^{\prime}$ by insertion and some other $n$-ary relations that contain members of $L^{\prime}-L$ are adjoined to those in $S$, or various original $n$-ary relations in $S$ are extended by adding $n$ tuples that contain members from $L^{\prime}-L$. For both the trivial and nontrivial cases and with respect to the language $L^{\prime}$, it follows that $C \leq C^{\prime}$. In the trivial case, if $X \subset L^{\prime}$, then $C(X)=C^{\prime}(X)=C(X \cap L) \cup(X-L)$.

In practice, a practical logic system is a logic system defined for the subsets of a finite language $L^{f}$. When a specific deduction is made from a set of hypotheses $X$, the set $X$ is finite. If the logic system also includes 1-ary sets, such as the logical or physical axioms, the actual set of axioms that might be used for a deduction is also finite. Indeed, the actual set of all deductions obtained at any moment in human history and used by a science community form a finite set of statements that are contained in a finite language $L^{f}$. (Finite languages, the associated consequence operators, and the like will usually be denoted by a superscript $f$.) The finitely many $n$-ary relations that model the rules of inference for a practical logic system are finite sets.

Practical logic systems generate practical consequence operators, and practical consequence operators generate effectively practical logic systems in many ways. For example, the method found in [7], when applied to a $C^{f}$, will effectively generate a finite set of rules of inference. The practical logic system obtained from such rules generates the original practical consequence operator. Hence, a consequence operator $C^{f}$ defined on $L^{f}$ is considered a practical consequence operator although it may not correspond to a previously defined scientific practical logic system; nevertheless, it does correspond to an equivalent practical logic system.

Our definition of a physical theory is a refinement of the usual definition. Given a set of physical hypotheses, general scientific statements are deduced. If accepted by a science community, these statements become natural laws. These natural laws then become part of a science-community logic system.

In [5], a consequence operator generated by such a logic system is denoted by $S_{N}$. From collections of such logic systems, the $S_{N}$ they generate are then applied to specific natural-system descriptions $X$. For scientific practical logic systems, the language and rules of inference need not be completely determinate in that, in practice, the language and rules of inference are extended.

The complete Tarski definition for a consequence operator includes finite languages [8, page 63] and all Tarski results used in this paper apply to such finite languages. Theorem 2.10 holds for any language, finite or not. In the lattice of finitary consequence operators defined on $L^{f}, \vee_{w}$ determines the least upper bound for a finite set of such operators. However, it is certainly possible that this least upper bound is the upper unit $U$. 
DEFINITION 3.1. Let $C$ be a general consequence operator defined in $L$. Let $X \subset L$.

(i) The set $X$ is $C$-consistent if $C(X) \neq L$.

(ii) The set $X$ is $C$-complete if for each $x \in L$, either $x \in X$ or $C(X \cup\{x\})=L$.

(iii) A set $X \subset L$ is maximally $C$-consistent if $X$ is $C$-consistent and whenever a set $Y \neq X$ and $X \subset Y \subset L$, then $C(Y)=L$.

Notice that if $X \subset L$ is $C$-consistent, then $C(X)$ is a $C$-consistent extension of $X$ which is also a $C$-system. Further, $C$-consistent $W$ is $C$-consistent with respect to any trivial extension of $C$ to a language $L^{\prime} \supset L$.

THEOREM 3.2. Let the general consequence operator $C$ be defined on $L$.

(i) The set $X \subset L$ is $C$-complete and $C$-consistent if and only if $X$ is maximally $C$ consistent.

(ii) If $X$ is maximally $C$-consistent, then $X$ is a $C$-system.

Proof. (i) Let $X$ be maximally $C$-consistent. Then $X$ is $C$-consistent and, hence, $C(X) \neq L$. Hence, let $x \in L$ and $x \notin X$. Then $X \subset X \cup\{x\}$ implies that $X \cup\{x\}$ is not $C$-consistent. Thus $C(X \cup\{x\})=L$. Hence, $X$ is $C$-complete. Conversely, assume that $X$ is $C$-consistent and $C$-complete. Then $X \neq L$. Let $X \subset Y \subset L$ and $X \neq Y$. Hence, there is some $y \in Y-X$ and from $C$-completeness, $L=C(X \cup\{y\}) \subset C(Y)$. Thus, $Y$ is not $C$-consistent. Hence, $X$ is maximally $C$-consistent and the result follows.

(ii) From $C$-consistency, $C(X) \neq L$. If $x \in C(X)-X$, then maximal $C$-consistency implies that $L=C(X \cup\{x\}) \subset C(C(X))=C(X)$. This contradiction yields that $X$ is a C-system.

The following easily obtained result holds for many types of languages [8, page 98], but these "Lindenbaum" constructions, for infinite languages, are not considered effective. For finite languages, such constructions are obviously effective.

THEOREM 3.3. Let the practical consequence operator $C^{f}$ be defined on arbitrary $L^{f}$. If $X \subset L^{f}$ is $C^{f}$-consistent, then there exists an effectively constructed $Y \subset L^{f}$ such that $C^{f}(X) \subset Y$ and $Y$ is $C^{f}$-consistent and $C^{f}$-complete.

Proof. This is rather trivial for a practical consequence operator, and all of the construction processes are effective. Consider an enumeration for $L^{f}$ such that $L^{f}=$ $\left\{x_{1}, x_{2}, \ldots, x_{k}\right\}$. Let $X \subset L^{f}$ be $C^{f}$-consistent and define $X=X_{0}$. We now simply construct in a completely effective manner a partial sequence of subsets of $L^{f}$. Simply consider $X_{0} \cup\left\{x_{1}\right\}$. Since $X_{0}$ is $C^{f}$-consistent, we have two possibilities. Effectively determine whether $C^{f}\left(X_{0} \cup\left\{x_{1}\right\}\right)=L^{f}$. If so, let $X_{1}=X_{0}$. On the other hand, if $C^{f}\left(X_{0} \cup\left\{x_{1}\right\}\right) \neq L^{f}$, then define $X_{1}=X_{0} \cup\left\{x_{1}\right\}$. Repeat this construction finitely many times. (Usually, if the language is denumerable, this is expressed in an induction format.) Let $Y=X_{k}$. By definition, $Y$ is $C^{f}$-consistent. Suppose that $x \in L^{f}$. Then there is some $X_{i}$ such that either (a) $x \in X_{i}$ or (b) $C^{f}\left(X_{i} \cup\{x\}\right)=L^{f}$. For (a), since $X_{i} \subset Y, x \in Y$. For (b), $X_{i} \subset Y$ implies that $L=C^{f}\left(X_{i} \cup\{x\}\right) \subset C^{f}(Y \cup\{x\})=L^{f}$. Hence, $Y$ is $C^{f}$-complete and $X_{i} \subset Y$, for each $i=1, \ldots, k$. By Theorem 3.2, $Y$ is a $C^{f}$-system. Thus $X_{0} \subset Y$ implies that $C^{f}\left(X_{0}\right) \subset C^{f}(Y)=Y$, and this completes the proof.

COROLlary 3.4. Let a practical consequence operator $C^{f}$ be defined on $L^{f}$ and let $X \subset L^{f}$ be $C^{f}$-consistent. Then there exists an effectively constructed $Y \subset L^{f}$ that is an 
extension of $C^{f}(X)$ and, hence, also an extension of $X$, where $Y$ is a maximally $C^{f}$ consistent $C^{f}$-system.

Let the set $\Sigma^{p} \subset \Sigma$ consist of all of the science-community practical logic systems defined on languages $L_{i}^{f}$. Each member of $\Sigma^{p}$ corresponds to $i \in\left|\Sigma^{p}\right|$ and to a practical consequence operator $C_{i}^{f}$ defined on $L_{i}^{f}$. In general, the members of a set of sciencecommunity logic systems are related by a consistency notion relative to an extended language.

Definition 3.5. A set of consequence operators $\mathscr{C}$ defined on $L$ is system consistent if there exists $Y \subset L, Y \neq L$, and $Y$ is a $C$-system for each $C \in \mathscr{C}$.

EXAMPLE 3.6. Let $\mathscr{C}$ be a set of axiomless consequence operators where each $C \in \mathscr{C}$ is defined on $L$. In [5], the set of science-community consequence operators is redefined by relativization to produce a set of axiomless consequence operators, $S_{N}^{V}$, each defined on the same language. Any such collection $\mathscr{C}$ is system consistent since for each $C \in$ $\mathscr{b}, C(\varnothing)=\varnothing \neq L$.

EXAMPLE 3.7. One of the major goals of certain science communities is to find what is called a "grand unification theory." This is actually a theory that will unify only the four fundamental interactions (forces). It is then claimed that this will somehow lead to a unification of all physical theories. Undoubtedly, if this type of grand unification is achieved, all other physical science theories will require some type of restructuring. The simplest way this can be done is to use informally the logic-system expansion technique. This will lead to associated consequence operators defined on "larger" language sets.

Let a practical logic system $S_{0}$ be defined on $L_{0}^{f}, L=\bigcup\left\{L_{i}^{f} \mid i \in \mathbb{N}\right\}$, with $\mathbb{N}$ the set of natural numbers. Let $L_{0} \subset L_{1}, L_{0} \neq L_{1}$. (Note that the remaining members of $\left\{L_{i}^{f} \mid i \in \mathbb{N}\right\}$ need not be distinct.) Expand $S_{0}$ to $S_{1} \neq S_{0}$ defined on $L$ by adjoining to the logic system $S_{0}$ finitely many practical logic-system $n$-ary relations or finitely many additional $n$ tuples to the original $S_{0}$, but where all of these additions only contain members from nonempty $L-L_{0}^{f}$. Although $S_{1}$ should only be considered as nontrivially defined on $L_{1}^{f}$, if $L \neq L_{1}$, then the $S_{1}$ so obtained corresponds to $C_{1}$, a consequence operator trivially extended to $L$. This process can be repeated in order to produce, at least, finitely many distinct logic systems $S_{i}, i>1$, that extend $S_{0}$ and a set $\mathscr{C}_{1}$ of distinct corresponding consequence operators $C_{i}$.

Since these are science-community logic systems, there is an $X_{0} \subset L_{0}^{f}$ that is $C_{0}^{f}$ consistent. By Corollary 3.4, there is an effectively defined set $Y \subset L_{0}^{f}$ such that $X_{0} \subset Y$ and $Y$ is maximally $C_{0}^{f}$-consistent with respect to the language $L_{0}^{f}$. Hence, $C_{0}^{f}(Y)=$ $Y \subset L_{0}^{f}$ and $C_{0}^{f}(Y) \neq L_{0}^{f}$. Further, $C_{0}^{f}$ is considered trivially extended to $L$. Let $Y^{\prime}=$ $Y \cup\left(L-L_{0}^{f}\right)$. It follows that for each $C_{i}, L-L_{0}^{f} \subset C_{i}\left(L-L_{0}^{f}\right) \subset L-L_{0}^{f} \neq L$. By construction, for each $C_{i}, C_{i}(Y)=Y$; and for each $X \subset L, C_{i}(X)=C_{0}\left(X \cap L_{0}^{f}\right) \cup C_{i}\left(X \cap\left(L-L_{0}^{f}\right)\right)$. So, let $X=Y^{\prime}$. Then for each $C_{i}, C_{i}\left(Y^{\prime}\right)=C_{0}(Y) \cup\left(L-L_{0}^{f}\right)=Y \cup\left(L-L_{0}^{f}\right)=Y^{\prime} \neq L$. Hence, the set of all $C_{i}$ is system consistent.

EXAMPLE 3.8. Consider a denumerable language $L$ and [2, Example 3.2]. (Note that there is a typographical error in this example. The expression $x \notin U$ should read $x \notin U$.) 
Let $U$ be a free ultrafilter on $L$ and let $x \in L$. Then there exists some $U \in U$ such that $x \notin$ $U$ since $\cap U=\varnothing$ and $\varnothing \notin U$. Let $B=\{x\}$ and $\mathscr{C}=\{P(U, B) \mid U \in U\}$, where $P(U, B)$ is the finitary consequence operator defined by $P(U, B)(X)=U \cup X$ if $x \in X$ and $P(U, B)(X)=$ $X$ if $x \notin X$. (Note that this is the same operator $P$ that appears in the proof of [5, Theorem 6.4].) There exists, at least, a sequence $S=\left\{U_{i} \mid i \in \mathbb{N}\right\}$ such that $U_{0}=U$ and $U_{i+1} \subset$ $U_{i}, U_{i+1} \neq U_{i}$. It follows immediately from the definition that $P\left(U_{i+1}, B\right) \leq P\left(U_{i}, B\right)$ and $P\left(U_{i+1}, B\right)(B)=U_{i+1} \cup B \subset U_{i} \cup B$ for each $i \in \mathbb{N}$. Hence, in general, $P\left(U_{i+1}, B\right)<P\left(U_{i}, B\right)$ for each $i \in \mathbb{N}$. Let $Y=L-\{x\}$. Then $P\left(U_{i}, B\right)(Y)=U_{i} \cup(L-\{x\})=L-\{x\}=Y, i \in \mathbb{N}$. Thus, the collection $\left\{P\left(U_{i}, B\right) \mid i \in \mathbb{N}\right\}$ is system consistent.

THEOREM 3.9. Consider $\mathscr{A} \subset \mathscr{C}_{f}$ defined on $L$ and the $(\leq)$ least upper bound $\bigvee_{w} \mathscr{A}$. Then $\bigvee_{w} \mathscr{A} \in \mathscr{C}_{f}$, and if $\mathscr{A}$ is system consistent, then there exists some $Y \subset L$ such that $Y=\bigvee_{w} \mathscr{A}(Y)=C(Y) \neq L$ for each $C \in \mathscr{A}$ and $\bigvee_{w} \mathscr{A} \neq U$. Further, if $X \subset L, X \neq L$, is a $C$-system for each $C \in \mathscr{A}$, then $X=\bigvee_{w} \mathscr{A}(X)=C(X) \neq L$ for each $C \in \mathscr{A}$.

Proof. Corollary 2.11 yields the first conclusion. From the definition of system consistent, there exists some $Y \subset L$ such that $C(Y)=Y \neq L$ for each $C \in \mathscr{A}$. From Lemma 2.6, for each $C \in \mathscr{A}, \bigvee_{w} \mathscr{A}(Y)=C(Y) \neq L$. Hence, $\bigvee_{w} \mathscr{A} \neq U$. The last part of this theorem follows from Lemma 2.6 and the fact that $X$ is also a $\bigvee_{w} \mathscr{A}$-system. This completes the proof.

4. An ultralogic unification. Assume for nonempty $\Sigma$ that $|\Sigma| \leq \kappa_{0}$. Let $\mathscr{C}$ denote a set of (logic-system) corresponding finitary consequence operators, each considered as defined on the language $L$. There exists a surjection $f: \mathbb{N} \rightarrow \mathscr{b}$ such that $f(i)$ is one of the members of $\mathscr{C}$, and for each $C \in \mathscr{C}$, there is some $j \in \mathbb{N}$ such that $f(j)=C$. For each $i \in \mathbb{N}$, let $f(i)=C_{i}$ denote the consequence operators in $\mathscr{C}$. As usual, for the following theorem, we use the boldface type convention [4, page 21$]$, and for the case $\mathscr{C}, \underline{\mathscr{C}}$ will denote boldface type.

TheOREM 4.1. Let $L$ and $\left\{C_{i} \mid i \in \mathbb{N}\right\}=\mathscr{C}$ be defined as above. Suppose that every (nonempty) finite subset of $\mathscr{C}$ is system consistent.

(i) Then there exists a hyperfinite ultralogic $u \in * \mathscr{C}_{\mathbf{f}}$ defined on the set of all internal subsets of ${ }^{*} \mathbf{L}$ such that $\boldsymbol{U} \neq{ }^{*} \mathbf{U}$, and an internal $W \subset{ }^{*} \mathbf{L}$ such that, for each $C_{i} \in \mathscr{b}$, ${ }^{*} \mathbf{C}_{i}(W)=\boldsymbol{U}(W)=W \neq{ }^{*} \mathbf{L}$, where $\boldsymbol{U}(W) \subset{ }^{*} \mathbf{L}$.

(ii) For each internal $Y \subset{ }^{*} \mathbf{L}, \bigcup\left\{{ }^{*} \mathbf{C}_{i}(Y) \mid i \in \mathbb{N}\right\} \subset \boldsymbol{U}(Y) \subset{ }^{*} \mathbf{L}$.

(iii) If finite $X \subset L$, then $\bigcup\left\{{ }^{*} \mathbf{C}_{i}(\mathbf{X}) \mid i \in \mathbb{N}\right\} \subset \mathcal{U}(\mathbf{X})$, and if each member of $\mathscr{b}$ is a practical consequence operator, then $\bigcup\left\{\mathbf{C}_{i}(\mathbf{X}) \mid i \in \mathbb{N}\right\} \subset \mathcal{U}(\mathbf{X})$.

(iv) Let $X \subset L, X \neq L$, be a $C$-system for each $C \in \mathscr{C}$. Then ${ }^{*} \mathbf{X}={ }^{*} \mathbf{C}_{i}\left({ }^{*} \mathbf{X}\right)=\boldsymbol{U}\left({ }^{*} \mathbf{X}\right) \neq{ }^{*} \mathbf{L}$ for each $i \in \mathbb{N}$. If $X$ is finite, $\mathbf{X}={ }^{*} \mathbf{C}_{i}(\mathbf{X})=u(\mathbf{X})$ for each $i \in \mathbb{N}$. If for $j \in \mathbb{N}, C_{j}$ is a practical consequence operator, then $\mathbf{X}=\mathbf{C}_{j}(\mathbf{X})=\boldsymbol{U}(\mathbf{X})=\boldsymbol{u}(\mathbf{X})$.

Proof. Let $\left\langle\mathscr{C}_{f}, \vee_{w}, \wedge, I, U\right\rangle$ be the lattice of all finitary consequence operators defined on $L$. Consider this lattice, all of our intuitive consequence operators, our $L$, and all other defined objects to be embedded into the Grundlagen structure 9 [4]. Hence, they are embedded, in the usual manner, into the superstructure model $M=\langle\mathcal{N}, \in,=\rangle$ for all bounded formal expressions and this is further embedded into the superstructure 
y that contains a nonstandard elementary extension $* \mathcal{M}=\left\langle{ }^{*} \mathcal{N}, \in,=\right\rangle$ of the embedded $M$. Notice that from our identifications, any standard $\mathbf{X} \subset \mathbf{L}$ has the property that ${ }^{\sigma} \boldsymbol{X}=\boldsymbol{X}$, and if $\boldsymbol{X}$ is finite, then ${ }^{*} \boldsymbol{X}=\boldsymbol{X}$. Under our basic embedding, let $\boldsymbol{g}: \mathbb{N} \rightarrow \underline{\mathscr{C}}$ be a surjection in $\mathcal{N}$ that corresponds to $f$. Now consider the surjection ${ }^{*} g:{ }^{*} \mathbb{N} \rightarrow \underline{*}$. Let constant $a \in \mathbb{N}$. Under our special Grundlagen embedding procedures, $*(g(a))=$ $* g(* a)={ }^{*} \boldsymbol{g}(a)={ }^{*} \mathbf{C}_{a}$. Since ${ }^{*} \boldsymbol{g}$ is a surjection, an $a \in \mathbb{N}$ corresponds to a member of $\sigma_{\underline{G}}$ and vice versa. Thus, ${ }^{*} \boldsymbol{g}$ restricted to members of ${ }^{\sigma} \mathbb{N}=\mathbb{N}$ yields the entire set ${ }^{\sigma} \underline{\mathscr{C}}$.

Let nonempty $K \subset \mathscr{P}(L)$ be the set of all $X \neq L$ that if $X \in K$, then $X$ is a $C$-system for each $C \in \mathscr{C}$. By Theorem 3.9, the definitions and the properties of the lattice structure on $\mathscr{C}_{f}$, for clarity, the unsimplified and redundantly expressed sentences

$$
\begin{gathered}
\forall x\left(( x \neq \varnothing ) \wedge ( x \in \mathscr { F } ( \mathbb { N } ) ) \longrightarrow \exists y \exists w _ { 1 } \left(\left(y \in \mathscr{C}_{\mathbf{f}}\right) \wedge(y \neq \mathbf{U}) \wedge\left(w_{1} \in \mathscr{P}(\mathbf{L})\right) \wedge\right.\right. \\
\left(\forall z _ { 1 } \forall v _ { 1 } \forall v _ { 2 } \left(( v _ { 1 } \in x ) \wedge ( v _ { 2 } \in \mathbf { K } ) \wedge ( v _ { 1 } \in \mathbb { N } ) \wedge ( z _ { 1 } \in x ) \wedge ( z _ { 1 } \in \mathbb { N } ) \longrightarrow \left(g\left(z_{1}\right)\left(v_{2}\right)=\right.\right.\right. \\
\left.\left.\left.y\left(v_{2}\right)=v_{2} \subset \mathbf{L}\right) \wedge\left(y\left(v_{2}\right) \neq \mathbf{L}\right)\right)\right) \wedge(\forall v((v \in x) \wedge(v \in \mathbb{N}) \longrightarrow \\
\left.\left.g(v)\left(w_{1}\right)=y\left(w_{1}\right)=w_{1} \neq \mathbf{L}\right)\right) \wedge \forall z((z \in x) \wedge(z \in \mathbb{N}) \longrightarrow((g(z) \leq y) \wedge \\
\left.\left.\left.\forall w\left(\left(w \in \mathscr{C}_{\mathbf{f}}\right) \wedge \forall z_{1}\left(\left(z_{1} \in x\right) \wedge\left(z_{1} \in \mathbb{N}\right) \wedge\left(g\left(z_{1}\right) \leq w\right)\right) \longrightarrow(y \leq w)\right)\right)\right)\right), \\
\forall x \forall y\left(\left(x \in \mathscr{C}_{\mathbf{f}}\right) \wedge\left(y \in \mathscr{C}_{\mathbf{f}}\right) \longrightarrow((y \leq x) \longleftrightarrow\right. \\
\forall w((w \in \mathscr{P}(\mathbf{L})) \longrightarrow(y(w) \subset x(w)))),
\end{gathered}
$$

hold in $\mathcal{M}$. Hence, they hold under $*$-transfer in $* \mathcal{M}$ for objects in $* \mathcal{N}$. (Note that it is usually assumed that formal statements such as (4.1) can be made within a formal first-order language rather than expressing them explicitly.)

The set $* \mathscr{C}_{\mathrm{f}}$ is a collection of hyperfinite consequence operators, each defined on the internal subsets of ${ }^{*} \mathbf{L}$. Let infinite $\lambda \in *^{*} \mathbb{N}-\mathbb{N}$. Then $* g[[0, \lambda]]$ is a hyperfinite subset of $* \underline{\mathscr{C}} \subset * \mathscr{C}_{\mathbf{f}}$. Hence, from $*$-transformed sentences (4.1), there exists some hyperfinite $u \in{ }^{*} \mathscr{C}_{\mathbf{f}}$ defined on the set of all internal $Y \subset{ }^{*} \mathbf{L}$ with the properties that ${ }^{*} g(i)(Y) \subset \mathcal{U}(Y)$ for each $i \in[0, \lambda]$ and, in particular for $i \in \mathbb{N}$. Hence, $\bigcup\left\{{ }^{*} \mathbf{C}_{i}(Y) \mid\right.$ $i \in \mathbb{N}\} \subset \mathcal{U}(Y)$. Further, there exists an internal $W \subset{ }^{*} \mathbf{L}$ such that, for each $i \in[0, \lambda]$, ${ }^{*} g(i)(W)=\mathcal{U}(W)=W \neq{ }^{*} \mathbf{L}$. In particular, ${ }^{*} g(i)(W)={ }^{*} \mathbf{C}_{i}(W)=\mathcal{U}(W)$ for each $i \in \mathbb{N}$. Since $U(W) \neq{ }^{*} \mathbf{L}$, then $\boldsymbol{U} \neq{ }^{*} \mathbf{U}$. Let finite $X \subset L$. Then, due to our embedding procedures, ${ }^{*}\left(\mathbf{C}_{i}(\mathbf{X})\right)={ }^{*} \mathbf{C}_{i}\left({ }^{*} \mathbf{X}\right)={ }^{*} \mathbf{C}_{i}(\mathbf{X}) \subset \boldsymbol{U}(\mathbf{X}) \neq{ }^{*} \mathbf{L}$. Hence, $\bigcup\left\{{ }^{*} \mathbf{C}_{i}(\mathbf{X}) \mid i \in \mathbb{N}\right\} \subset \boldsymbol{U}(\mathbf{X})$. (Note that in proofs such as this and to avoid confusion, we often, at first, use the notation $*\left(\mathbf{C}_{i}(\mathbf{X})\right)$ to indicate the value (or name) of the result of applying $*$ to an object in $\mathcal{N}$ which is a set such as $\mathbf{C}_{i}(\mathbf{X})$ that contains additional operator notation. From a technical viewpoint, ${ }^{*}\left(\mathbf{C}_{i}(\mathbf{X})\right)={ }^{*}\left\{\mathbf{C}_{i}(\mathbf{X})\right\}=\left\{{ }^{*} \mathbf{C}_{i}(\mathbf{X})\right\}$ and ${ }^{*} \mathbf{C}_{i}(\mathbf{X})$ is the "name" for the set under the mapping $*$. But using this procedure, there is confusion as to whether $*\left(\mathbf{C}_{i}(\mathbf{X})\right)$ denotes the entire set or denotes the operator ${ }^{*} \mathbf{C}_{i}$ applied to $\mathbf{X}$. In these proofs, ${ }^{*} \mathbf{C}_{i}$ always denotes the operator ${ }^{*} \mathbf{C}_{i}$ applied to internal subsets of ${ }^{*} \mathbf{L}$.) If $C_{i}$ is a practical consequence operator, then $C_{i}(X)$ is a finite set. Hence, ${ }^{*} \mathbf{C}_{i}(\mathbf{X})=\mathbf{C}_{i}(\mathbf{X})$. (iv) follows by *-transfer and this completes the proof. 
In [5], the set $\mathscr{S}=\left\{S_{N_{j}}^{V} \mid j \in \mathbb{N}\right\}$ is the refined set of all relativized axiomless sciencecommunity consequence operators defined on a language $\Lambda$ and they are used to unify, in a restricted manner, physical theory behavior. Moreover, for sequentially presented $\left\{S_{N_{j}}^{V} \mid j \in \mathbb{N}\right\}, 1 \leq\left|\left\{S_{N_{j}}^{V} \mid j \in \mathbb{N}\right\}\right| \leq \mathbb{N}_{0}$.

Corollary 4.2. Let $\Lambda$ denote the language $L$ and $\mathscr{S}=\left\{S_{N_{j}}^{V} \mid j \in \mathbb{N}\right\}$.

(i) There exists a hyperfinite ultralogic $U \in *^{*} \mathscr{C}_{\mathrm{f}}$ that is defined on all internal $Y \subset * \Lambda$ such that $u \neq * \mathbf{U}$, and, for each $S_{N_{i}}^{V} \in \mathscr{Y},{ }^{*} \mathbf{S}_{\mathrm{N} i}^{\mathrm{V}}(\varnothing)=u(\varnothing)=\varnothing$.

(ii) For each internal $Y \subset{ }^{*} \Lambda, \bigcup\left\{{ }^{*} \mathbf{S}_{\mathbf{N} i}^{\mathrm{V}}(Y) \mid i \in \mathbb{N}\right\} \subset \mathcal{U}(Y) \subset * \Lambda$.

(iii) If finite $X \subset \Lambda$, then $\bigcup\left\{{ }^{*} \mathbf{S}_{\mathbf{N} i}^{\mathrm{V}}(\mathbf{X}) \mid i \in \mathbb{N}\right\} \subset \mathcal{U}(\mathbf{X})$, and if each member of $\left\{S_{N_{i}}^{V} \mid i \in\right.$ $\mathbb{N}\}$ is a practical consequence operator, then $\bigcup\left\{\mathbf{S}_{\mathbf{N} i}^{\mathrm{V}}(\mathbf{X}) \mid i \in \mathbb{N}\right\} \subset \mathcal{U}(\mathbf{X})$.

(iv) Let $X \subset \Lambda, X \neq \Lambda$, be a C-system for each $C \in\left\{S_{N i}^{V}(X) \mid i \in \mathbb{N}\right\}$. Then ${ }^{*} \mathbf{X}=$ ${ }^{*} \mathbf{S}_{\mathbf{N} i}^{\mathbf{V}}\left({ }^{*} \mathbf{X}\right)=\boldsymbol{U}\left({ }^{*} \mathbf{X}\right) \neq * \Lambda$, for each $i \in \mathbb{N}$. If $X$ is finite, then ${ }^{*} \mathbf{S}_{\mathbf{N} i}^{\mathbf{V}}(\mathbf{X})=\mathbf{X}=\boldsymbol{U}(\mathbf{X})$ for each $i \in \mathbb{N}$. If for $i \in \mathbb{N}, S_{N_{i}}^{V}$ is a practical consequence operator, then $\mathbf{S}_{\mathbf{N} i}^{\mathrm{V}}(\mathbf{X})=\mathbf{X}=\boldsymbol{U}(\mathbf{X})$.

5. Further applications. If the $\mathscr{C}$ in the hypotheses of Theorem 4.1 is restricted to a set of practical consequence operators, each defined on a finite language $L^{f}$, then it follows that the hyperfinite $U$ corresponds to a hyperfinite logic system $\mathscr{S}$ that is $*$-effectively $*$-generated. If the effective notion is not required, then, in general, the ultralogic $u$ corresponds to a $*$-logic system. Although Theorem 4.1 and Corollary 4.2 are mainly concerned with the original set of consequence operators $\mathscr{C}$ and $\left\{S_{N_{i}}^{V}(X)\right.$ | $i \in \mathbb{N}\}$, when $\left|\left\{S_{N_{i}}^{V}(X) \mid i \in \mathbb{N}\right\}\right|=\mathcal{N}_{0}$, it is also significant for applications that $\mathcal{U}$ unifies each "ultranatural relativized theory" $* g(j), j \in[0, \lambda]-\mathbb{N}$. It follows that for each $j \in[0, \lambda]-\mathbb{N},{ }^{*} g(j)(\varnothing)=u(\varnothing)=\varnothing$, and for internal $Y \subset{ }^{*} \Lambda,{ }^{*} g(j)(Y) \subset \mathcal{U}(W)$. This also applies to the unrelativized case with the modifications that $\varnothing$ is replaced with $W$ and each relativized consequence operator $S_{N_{i}}^{V}$ is replaced with the physical theory consequence operator $S_{N_{i}}$. Also note that $S_{N_{i}}$ and $S_{N_{i}}^{V}$ are usually considered practical consequence operators.

Depending upon the set $\mathscr{b}$ of consequence operators employed, there are usually many $X \subset L, X \neq L$, such that $X$ is a $C$-system for each $C \in \mathscr{C}$. For example, we assumed in [5] that there are two 1-ary relations for the science-community logic systems. One of these contains the logical axioms and the other contains a set of physical axioms; a set of natural laws. Let $\left\{S_{N_{i}}^{\prime} \mid i \in \mathbb{N}\right\}$ be the set of science-community corresponding consequence operators relativized so as to remove the set of logical theorems. Each member of a properly stated set of natural laws $N_{j}$ used to generate the consequence operators $\left\{S_{N_{i}}^{\prime} \mid i \in \mathbb{N}\right\}$ should be a $C$-system for each member of $\left\{S_{N_{i}}^{\prime} \mid i \in \mathbb{N}\right\}$. As mentioned, the physical theories being considered here are not theories that produce new "natural laws." The argument that the Einstein-Hilbert equations characterize gravitation fields, in general, leads to the acceptance by many science communities of these equations as a "natural law" that is then applied to actual physical objects. Newton's Second Law of Motion is a statement about the notion of inertia within our universe. It can now be derived from basic laboratory observation and has been shown to hold for other physical models distinct from its standard usage [3]. The logic systems that generate the members of $\left\{S_{N_{i}}^{\prime} \mid i \in \mathbb{N}\right\}$ have, as a 1-ary relation, a set of natural laws. Then one takes a set of specific physical hypotheses $X$ that describes the behavior of a natural 
system and applies the logic system to $X$. This gives a statement on how these natural laws affect, if at all, the behavior being described by $X$. It is this approach that implies that each properly described $N_{j} \neq L$ is a $C$-system for each $C \in\left\{S_{N_{i}} \mid i \in \mathbb{N}\right\}$. Applying Theorem 4.1 to $\mathscr{C}=\left\{S_{N_{i}}^{\prime} \mid i \in \mathbb{N}\right\}$, where $\bigcup\left\{N_{i} \mid i \in \mathbb{N}\right\} \subset K$, leads to a result exactly like Corollary 4.2, where results (i), (iii), and (iv) applied to members of $\left\{N_{i} \mid i \in \mathbb{N}\right\}$ are particularly significant.

At any moment in human history, one can assume, due to the parameters present, that there is, at least, a denumerable set of science-community logic systems or that there exists only a finite collection of practical logic systems defined on finite $L^{f}$. The corresponding set $\mathscr{C}^{f}=\left\{C_{i}^{f} \mid i=1, \ldots, n\right\} \subset \mathscr{C}_{f}^{f}$ of practical consequence operators would tend to vary in cardinality at different moments in human history. For the corresponding finite set of practical consequence operators, by Theorem 2.10, there is a standard (least upper bound) practical consequence operator $u$, and hence, "the best" practical logic system, that unifies such a finite set. The following result is the interpretation of Theorem 4.1 for such a finite set of practical consequence operators.

THEOREM 5.1. Let $L^{f}$ and $\mathscr{C}^{f}$ be defined as above. Suppose that $\mathscr{C}^{f}$ is system consistent.

(i) Then there exists a practical consequence operator $u_{1} \in \mathscr{C}_{f}^{f}$ defined on the set of all subsets of $L^{f}$ such that $\varkappa_{1} \neq U$, and $W \subset L$ such that, for each $C_{i} \in \mathscr{C}^{f}, C_{i}(W)=$ $u_{1}(W)=W \neq L^{f}$, where $u_{1}(W) \subset L^{f}$.

(ii) For each $X \subset L^{f}, \bigcup\left\{C_{i}(X) \mid i \in \mathbb{N}\right\} \subset \cup_{1}(X) \subset L^{f}$ and $u_{1}$ is the least upper bound in $\left\langle\mathscr{C}_{f}^{f}, \vee_{w}, \wedge, I, U\right\rangle$ for $\mathscr{C}^{f}$.

(iii) Let $X \subset L^{f}, X \neq L^{f}$, be a $C$-system for each $C \in \mathscr{C}^{f}$. Then $X=C_{i}(X)=\mathcal{U}_{1}(X) \neq L^{f}$ for each $i \in \mathbb{N}$.

Letting finite $\mathscr{C}^{f}$ contain practical consequence operators of the type $S_{N_{i}}, S_{N_{i}}^{V}$, or $S_{N_{i}}^{\prime}$, exclusively, then $U_{1}$ would have the appropriate additional properties and would generate a practical logic system. Corollary 2.11 and Theorem 3.9 yield a more general unification $\bigvee_{w} \mathscr{A}, \mathscr{A} \subset \mathscr{C}_{f}$, as represented by a least upper bound in $\left\langle\mathscr{C}_{f}, \vee_{w}, I, U\right\rangle$, with the same properties as stated in Theorem 5.1. Thus, depending upon how physical theories are presented and assuming system consistency, there are nontrivial standard unifications for such physical theories. Assuming that $|\mathscr{A}|=\aleph_{0}$ and that $\mathscr{A}$ is system consistent, then the Corollary 2.11 unification $\bigvee_{w} \mathscr{A}$ corresponds to a (nontrivial) nonstandard ultralogic unification ${ }^{*} \bigvee_{w} \mathscr{A}$ with all of the same stated properties as those of the $U$. It is obvious how these two ultralogic unifications model a higher intelligence relative to general intelligent design theory. However, ${ }^{*} \bigvee_{w} \mathscr{A}$ and $u$ have one significant difference. The ultralogic $\mathcal{U}$ is, with respect to internal subsets of $* \mathbf{L}$, a "least upper bound" of a hyperfinite collection, whereas $* \bigvee_{w} \mathscr{A}$ need not have this additional hyperfinite property. Further, system consistency is used only so that one statement in Theorem 4.1, Corollary 4.2, Theorem 5.1, and this paragraph will hold. This one fact is that each of these standard unifications of a collection $\mathscr{A} \subset \mathscr{C}_{f}$ is not the same as the upper unit if and only if $\mathscr{A}$ is system consistent. Further, if an $X \subset L^{f}$ (resp., $X \subset L$ ) is $\mathscr{U}_{1}$-consistent (resp., $\bigvee_{w} \mathscr{A}$-consistent), $X$ is $C$-consistent for each $C \in \mathscr{C}^{f}$ (resp., $C \in \mathscr{A}$ ). 
REMARK 5.2. I mentioned that the nonstandard results obtained in Section 4 are established by means of the most trivial methods used in Robinson-styled nonstandard analysis.

\section{REFERENCES}

[1] W. Dziobiak, The lattice of strengthenings of a strongly finite consequence operation, Studia Logica 40 (1981), no. 2, 177-193.

[2] R. A. Herrmann, Nonstandard consequence operators, Kobe J. Math. 4 (1987), no. 1, 1-14.

[3] _ Newton's second law of motion holds in normed linear spaces, Far East J. Appl. Math. 2 (1998), no. 3, 183-190.

[4] _ The Theory of Ultralogics. Part I, http://www.arXiv.org/abs/math.GM/9903081, 1999.

[5] _ Hyperfinite and standard unifications for physical theories, Int. J. Math. Math. Sci. 28 (2001), no. 2, 93-102.

[6] _ Ultralogics and probability models, Int. J. Math. Math. Sci. 27 (2001), no. 5, 321-325.

[7] J. Łoś and R. Suszko, Remarks on sentential logics, Indag. Math. 20 (1958), 177-183.

[8] A. Tarski, Logic, Semantics, Metamathematics. Papers from 1923 to 1938, Clarendon Press, Oxford, 1956.

[9] R. Wójcicki, Some remarks on the consequence operation in sentential logics, Fund. Math. 68 (1970), 269-279.

[10] - On matrix representations of consequence operations of Łukasiewicz's sentential calculi, Z. Math. Logik Grundlagen Math. 19 (1973), 239-247.

Robert A. Herrmann: Mathematics Department, United States Naval Academy, 572C Holloway Road, Annapolis, MD 21402-5002, USA

E-mail address: rah@usna.edu 


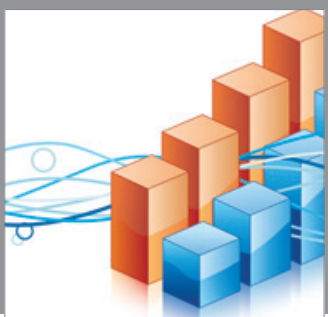

Advances in

Operations Research

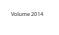

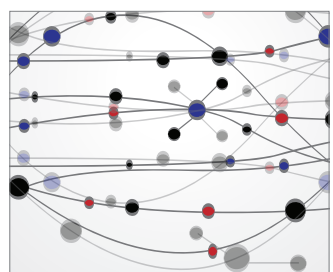

\section{The Scientific} World Journal
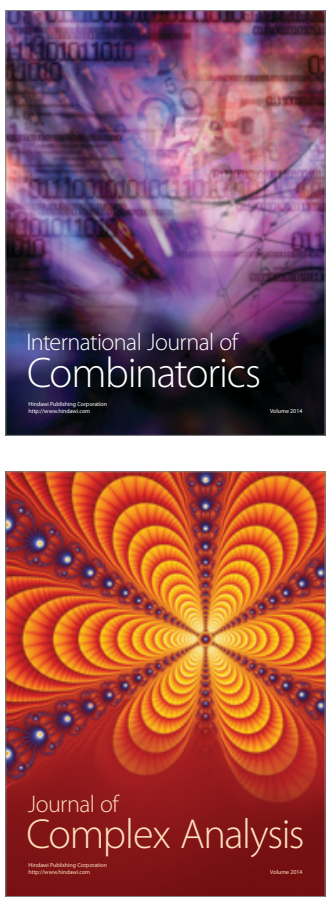

International Journal of

Mathematics and

Mathematical

Sciences
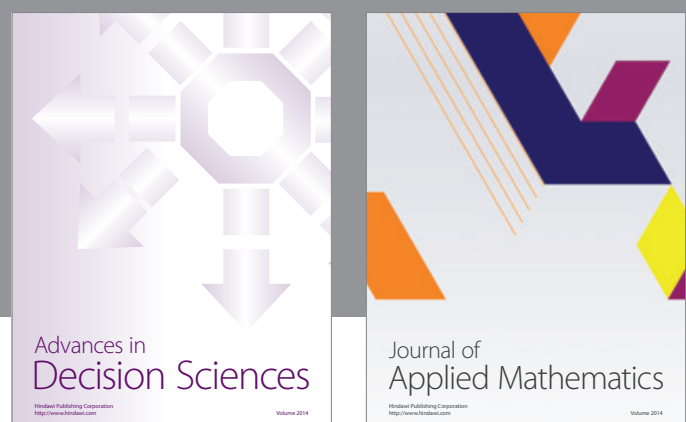

Journal of

Applied Mathematics
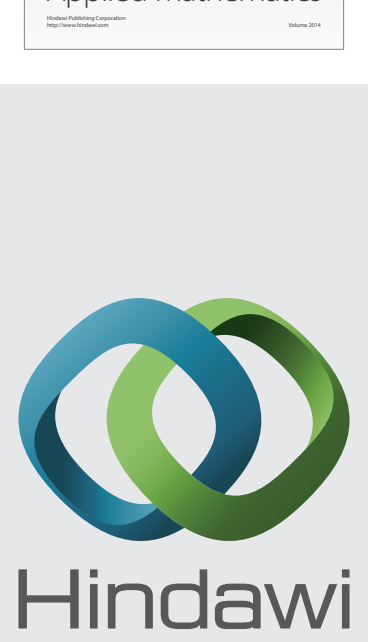

Submit your manuscripts at http://www.hindawi.com
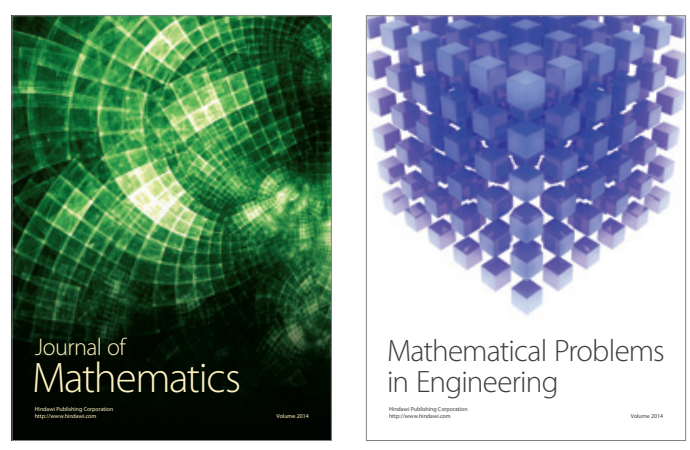

Mathematical Problems in Engineering
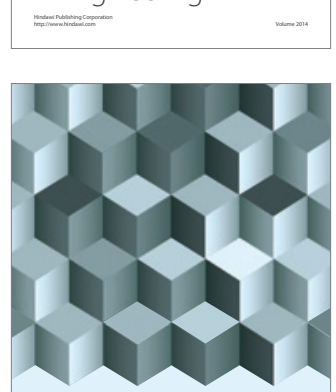

Journal of

Function Spaces
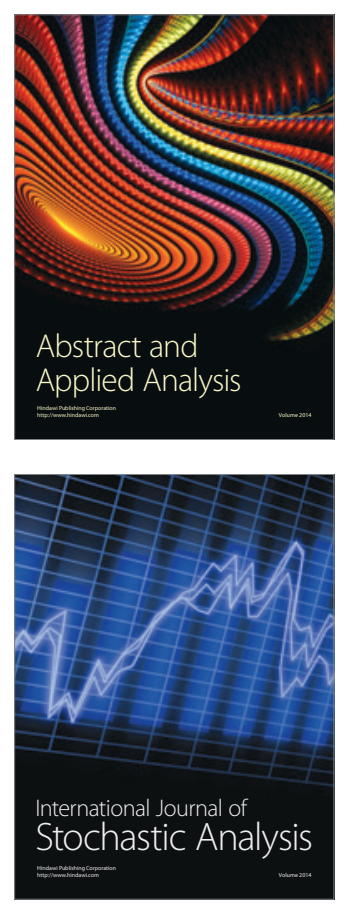

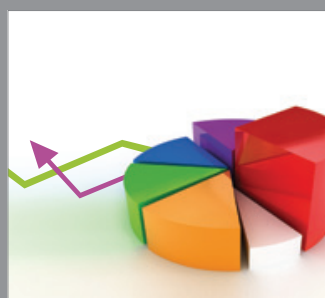

ournal of

Probability and Statistics

Promensencen
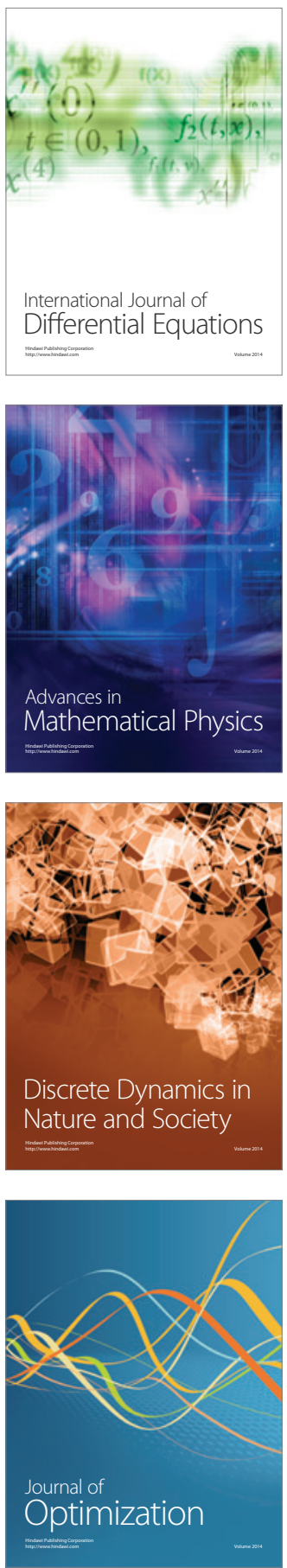